ISBN 978-93-86878-06-9

11th International Conference on Data Mining, Computers, Communication and Industrial

Applications (DMCCIA-2017)

Kuala Lumpur (Malaysia) Dec. 14-15, 2017

\title{
MQTT of IoT Classification in Energy Saving
}

\author{
Sujita Jiwangkura ${ }^{1}$, Peraphon Sophatsathit ${ }^{2}$ and Achara Chandrachai ${ }^{3}$ \\ ${ }^{1}$ Technopreneurship and Innovation Management Program, Graduate School, Chulalongkorn University, \\ Bangkok, Thailand \\ ${ }^{2}$ Department of Mathematics and Computer Science, Faculty of Science, Chulalongkorn University, \\ Bangkok, Thailand \\ ${ }^{3}$ Department of Commerce, Faculty of Commerce and Accountancy, Chulalongkorn University, \\ Bangkok, Thailand
}

\begin{abstract}
Industries today face the growing problems from scarce energy. Energy awareness will help industrial organizations survive in terms of availability and cost of energy. With IoT technology, MQTT serves as an application protocol for energy saving. However, there are few reviews on evidence of energy savings by MQTT of IoT after commercialization. The MQTT classification is proposed to identify MQTT energy technology and related technologies. This article surveys experimented MQTT application articles published between 2011 and 2017 to confirm this classification. Survey results indicate energy saving in all articles. The findings show that MQTT is a suitable IoT protocol for industrial applications that supports energy saving for industries.
\end{abstract}

Keywords: IoT, MQTT, Energy saving, Energy awareness

\section{Introduction}

The Internet of Things (IoT) is a megatrend. It changes the business world as a competitive requirement in the global industries to communicate different devices running applications in the network [27]. While several IoT application systems and frameworks have been developed by using MQTT protocol [23], the needs of MQTT technology to support the industries are mandatory in the development process. However, there are few reviews on the contribution of MQTT in energy savings. The objective of this paper is to propose IoT researchers and managers the classification of MQTT with the focus on energy considerations in industries. A critical survey of articles in MQTT published after commercialization in the last 7 years from leading publishers, such as Elsevier, Emerald, IEEE, Sage, Springer and Taylor Francis, is conducted to confirm the classification. The topics studied from the survey are MQTT application, platform technology, database technology, energy technology and technological industry. Analyses and the results are presented.

This paper is organized as follows. Section 2 surveys the MQTT application and technologies. Section 3 provides the analysis, results of surveyed articles and the proposed MQTT classification. Finally, Section 4 gives the conclusion.

\section{Survey of MQTT}

As MQTT has been commercialized since 2011, this survey deeply searches for industrial application and technologies of MQTT scientific articles, peer-reviewed and published between 2011 and 2017. Proceedings and books are avoided.

MQTT Application

Message Queuing Telemetry Transport (MQTT) is a protocol developed by Professor Dr. Andy StanfordClark of IBM and Arlen Nipper of Arcom in 1999 to handle messages for IoT devices [3]. MQTT uses 
telemetry technology to send and receive information from a distance over the internet in real-time by message [27]. It has been a standardized application protocol by OASIS standard since 2013 [23]. MQTT messaging mechanism consists of 3 components, namely, publisher, broker and subscriber. MQTT is Message Oriented Middleware (MOM) approach and it is used for one-to-many (1:M) mapping for Machine-to-Machine (M2M) communication as its broker can transmit messages from a publisher to one or many subscribers. Its ease of use comes from 4 simple commands: publish, subscribe, connect and disconnect.

MQTT protocol is primarily deployed with IoT applications in several industries. The applications are developed and implemented to utilize the benefits of MQTT [1-2, 4-14, 16-21, 24, 26, 28-36]. Messages of connected constrained devices are managed and handled by MQTT in thousands of large queues before transmission [10, 14, 28]. MQTT is lightweight as it has a small header between 1 to 4 bytes [8, 12]. MQTT provides long-lived connection with constrained devices while they are working [33]. It can work in high latency or unreliable networks due to the heterogeneity of devices [31]. MQTT applications have two types of body message [1, 9, 29, 32-33] and equipment message [2, 4-8, 10-14, 16-21, 24, 26, 28, 30-31, 34-36]. In short, MQTT is energy saving-oriented which involves in many technological domains.

\section{Platform Technology}

MQTT can work on platform technologies such as Cloud computing and Fog computing. Cloud computing is a shared-resource service defined by the US National Institute of Standard and Technology (NIST). It is a mature technology which provides storage, computing and networking for customer requirements [1-2, 4-10, 12$14,16-21,24,26,28-30,32-36]$. It enables IoT devices to transmit messages by MQTT in many aspects. The displacement is centralized, the user access is ubiquitous and the data storage is virtually unlimited [27]. Cloud computing has many service types for different purposes: Infrastructure as a Service (IaaS), Platform as a Service (PaaS) and Software as a Service ( $\mathrm{SaaS})$. IaaS is suitable for large companies who focus to control the system. PaaS is suitable for software developers who avoid the platform investment. SaaS is suitable for small companies who focus to get a good service. Fog computing is an extended cloud service introduced by CISCO. It is a new technology which provides storage, computing and networking to the edge of network for quicker analysis $[11,31]$. It enables IoT devices to transmit messages locally by MQTT in many aspects. The location is the edge, the latency is low and the analysis is real-time. Fog computing has as many service types as cloud computing. Thus, it gives several benefits to IoT users in case of emergency. The decision making is immediate and the correct action can be taken accordingly.

Database Technology

MQTT transmits device messages to the subscriber who will store those message data in the database. Generally, there are two types of databases in IoT: SQL database and NoSQL database [22]. SQL is a relational database introduced by Edgar Codd of IBM in 1970. SQL database is traditionally used in IoT applications such as MySQL and Oracle [1-2, 5-7, 9-13, 16, 18, 20-21, 24, 26, 29-32, 34-36]. The benefits are reliability, consistency and maturity. However, SQL database has some limitations to delay IoT applications [22, 33]. The read-write speed is slow. The data structure is a fixed and complicated table. The deployment machine is expensive and the system is centralized. These limitations have brought about NoSQL or Not only SQL as an alternative to database. It was introduced by Amazon in 2007 as a non-relational database which managed unstructured big data. NoSQL is newly used in some special IoT applications such as MongoDB and Redis [4, 8, $14,17,19,28,33]$. NoSQL has some benefits to enhance IoT applications. The read-write speed is fast. The data structure is unfixed for several types of big data such as image, email, line and facebook. The deployment machine is inexpensive and the system is distributed. Nonetheless, NoSQL has some issues of reliability, consistency and maturity.

\section{Energy Technology}

MQTT energy technology comes from MQTT models which are MQTT and MQTT-SN [15]. MQTT runs on TCP/IP network whereas MQTT-SN runs on Wireless Sensor Network (WSN). MQTT for Sensor Networks 
(MQTT-SN) or MQTT for Sensors (MQTT-S) is an extension of MQTT for better efficiency to WSN. It was developed and tested by Stanford-Clark team on the IBM WSN testbed in 2008 to be an option of MQTT which relied on UDP. MQTT-SN adds more commands than MQTT for gateway requests. Small IoT devices have limited power. MQTT is designed to provide energy saving technology of low CPU usage in the transmission with those power-limited devices [1-2, 4-7, 9-11, 14, 16-21, 26, 28-30, 32-36]. MQTT is experimented up to a thousand samples to prove energy saving. MQTT-SN is designed to provide more energy saving technology for support of sleeping clients, support of multiple gateways and lightweight implementation [8, 12-13, 24, 31]. Hence, both MQTT and MQTT-SN offer energy saving in various technological industries.

Technological Industry

MQTT has been used in a diverse set of technological industries. Most MQTT uses of industries are healthcare, robot and industry 4.0 [25]. Healthcare is initially proven as MQTT applications. Patients and the elderly use wearable sensor devices to send body messages to the medical office. Nurses and medical persons use MQTT to send messages for hospital services [1, 9, 11, 21, 29, 32-33]. Generally, robot is used to protect human from dangerous tasks. It can do more in MQTT applications of robot industry to send and receive messages with human and robot $[6,20]$. Industry 4.0 is a smart city which provides smart objects and smart systems anywhere for its citizens to have a better life. Those objects and systems can be used to provide smart information for citizens, factories and natural environment in real-time [4, 7, 10, 13, 17-19, 28, 30-31, 36]. Consequently, these intelligent industries make the world more convenient.

\section{Analysis and Results}

The surveyed articles are analyzed by MQTT application and technologies. The MQTT application is analyzed by development, implementation, body message and equipment message, while the MQTT technologies are classified by platform, database, energy and technological industry. Platform technology is analyzed by cloud computing and fog computing. Database technology is analyzed by SQL, NoSQL and others. Energy technology is analyzed by low CPU usage, support of sleeping clients, support of multiple gateways and lightweight implementation. Technological industry is analyzed by healthcare, robot, industry 4.0 and others. The results yield 30 articles during 2015-2017 publications.

Results in Summary

Results of the surveyed articles on MQTT energy saving in industries are shown in Fig 1 and Table I

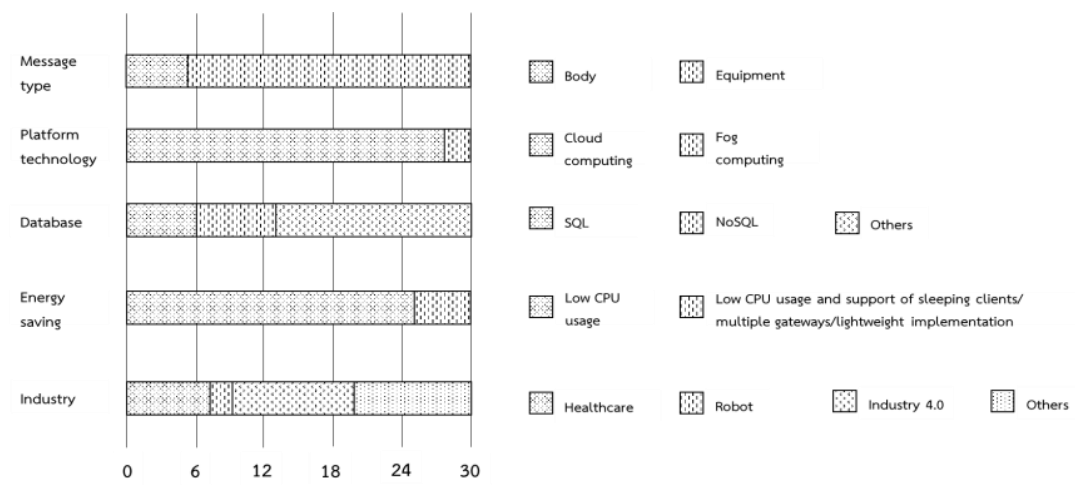

Fig. 1: Plots of resulting MQTT application and classification.

In Fig. 1, the analyzed results for message type consist of $16.67 \%$ body message and $83.33 \%$ equipment message. The results in platform technology consist of $93.33 \%$ cloud computing and $6.67 \%$ fog computing. The results in database technology consist of 20\% SQL, 23.33\% NoSQL and 56.67\% others. The results in energy technology consist of $83.33 \%$ low CPU usage, $16.67 \%$ low CPU usage and support of sleeping clients/multiple gateways/lightweight implementation. The results in technological industry consist of $23.33 \%$ healthcare, $6.67 \%$ robot, $36.67 \%$ industry 4.0 and $33.33 \%$ others. 
TABLE I: Articles Analyzed by MQTT Application and Classification

\begin{tabular}{|c|c|c|c|c|c|c|c|c|c|c|c|c|c|c|c|c|c|}
\hline & \multirow{2}{*}{\multicolumn{4}{|c|}{ MQTT Application }} & \multicolumn{13}{|c|}{ MQTT classification } \\
\hline & & & & & \multicolumn{2}{|c|}{$\begin{array}{l}\text { Platform } \\
\text { technology }\end{array}$} & \multicolumn{3}{|c|}{$\begin{array}{l}\text { Database } \\
\text { technology }\end{array}$} & \multicolumn{4}{|c|}{ Energy technology } & \multicolumn{4}{|c|}{$\begin{array}{l}\text { Technological } \\
\text { industry }\end{array}$} \\
\hline & 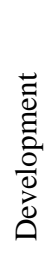 & 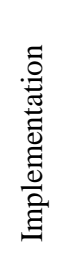 & $\begin{array}{l}2 \\
0 \\
0\end{array}$ & 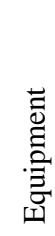 & 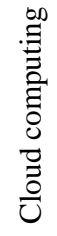 & 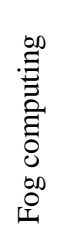 & $\ddot{\partial}$ & $\begin{array}{l}\partial \\
\tilde{z} \\
z\end{array}$ & $\frac{\mathscr{0}}{\tilde{0}}$ & 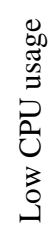 & 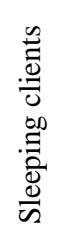 & 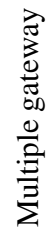 & 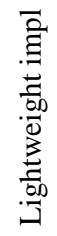 & 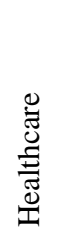 & $\begin{array}{l}\overrightarrow{0} \\
\stackrel{0}{0}\end{array}$ & $\begin{array}{l}0 \\
\dot{+} \\
\stackrel{2}{\Xi} \\
\stackrel{\Xi}{\Xi} \\
\stackrel{\Xi}{\Xi}\end{array}$ & 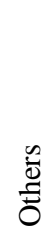 \\
\hline${ }^{2015}[1]$ & o & o & o & & 0 & & & & o & o & & & & o & & & \\
\hline${ }^{2015}[16]$ & ○ & ○ & & 0 & $\circ$ & & 0 & & & $\circ$ & & & & & & & 0 \\
\hline${ }^{2015}[19]$ & $\circ$ & $\circ$ & & $\circ$ & $\circ$ & & & 0 & & $\circ$ & & & & & & 0 & \\
\hline${ }^{2015}$ [20] & ० & ○ & & $\circ$ & ○ & & & & 0 & $\circ$ & & & & & $\circ$ & & \\
\hline${ }^{2015}[21]$ & $\circ$ & $\circ$ & & $\circ$ & $\circ$ & & & & $\circ$ & $\circ$ & & & & 0 & & & \\
\hline${ }^{2016}[10]$ & $\circ$ & $\circ$ & & $\circ$ & $\circ$ & & & & $\circ$ & $\circ$ & & & & & & 0 & \\
\hline${ }^{2016}[14]$ & $\circ$ & $\circ$ & & $\circ$ & $\circ$ & & & $\circ$ & & $\circ$ & & & & & & & ○ \\
\hline${ }^{2016}[24]$ & $\circ$ & ○ & & $\circ$ & ○ & & & & 0 & $\circ$ & 0 & 0 & 0 & & & & ○ \\
\hline${ }^{2016}[26]$ & $\circ$ & $\circ$ & & $\circ$ & $\circ$ & & & & ○ & $\circ$ & & & & & & & $\circ$ \\
\hline${ }^{2016}[28]$ & ० & o & & ○ & ○ & & & 0 & & ० & & & & & & 0 & \\
\hline${ }^{2016}[29]$ & $\circ$ & $\circ$ & $\circ$ & & $\circ$ & & ० & & & $\circ$ & & & & ० & & & \\
\hline${ }^{2016}[30]$ & ○ & o & & 0 & o & & o & & & ○ & & & & & & 0 & \\
\hline${ }^{2016}[31]$ & ० & ○ & & ○ & & 0 & & & 0 & ○ & 0 & ○ & 0 & & & $\circ$ & \\
\hline${ }^{2016}[33]$ & ○ & ○ & $\circ$ & & ○ & & & $\circ$ & & ○ & & & & o & & & \\
\hline${ }^{2016}[35]$ & ○ & ○ & & ○ & ○ & & ○ & & & ○ & & & & & & & ○ \\
\hline${ }^{2016}[36]$ & ○ & $\circ$ & & ○ & ○ & & & & 0 & ○ & & & & & & 0 & \\
\hline${ }^{2017}[2]$ & ○ & ○ & & ○ & ○ & & o & & & ○ & & & & & & & $\circ$ \\
\hline${ }^{2017}[4]$ & ० & ० & & ○ & ○ & & & 0 & & ○ & & & & & & 0 & \\
\hline${ }^{2017}[5]$ & ० & ○ & & $\circ$ & $\circ$ & & 0 & & & ○ & & & & & & & 0 \\
\hline${ }^{2017}[6]$ & ○ & ○ & & ○ & ○ & & & & ○ & $\circ$ & & & & & ○ & & \\
\hline${ }^{2017}[7]$ & ○ & ○ & & ○ & ○ & & & & ○ & ○ & & & & & & 0 & \\
\hline${ }^{2017}[8]$ & o & ○ & & ○ & ○ & & & 0 & & ○ & 0 & 0 & 0 & & & & $\circ$ \\
\hline${ }^{2017}[9]$ & ○ & $\circ$ & $\circ$ & & ○ & & & & o & $\circ$ & & & & o & & & \\
\hline${ }^{2017}[11]$ & ० & ० & & 0 & & 0 & & & ○ & ○ & & & & ० & & & \\
\hline $2017[12]$ & ○ & o & & ○ & ○ & & & & ○ & ○ & 0 & o & 0 & & & & 0 \\
\hline${ }^{2017}[13]$ & ○ & ○ & & $\circ$ & ○ & & & & ○ & ○ & ○ & ○ & ○ & & & 0 & \\
\hline${ }^{2017}[17]$ & ○ & ○ & & ○ & ○ & & & 0 & & ○ & & & & & & ○ & \\
\hline${ }^{2017}[18]$ & o & ○ & & ○ & ○ & & & & 0 & ○ & & & & & & ○ & \\
\hline${ }^{2017}[32]$ & ○ & ○ & 0 & & ○ & & & & ○ & ○ & & & & 0 & & & \\
\hline${ }^{2017}[34]$ & $\circ$ & ○ & & $\circ$ & $\circ$ & & & & $\circ$ & $\circ$ & & & & & & & 0 \\
\hline
\end{tabular}

\section{Results Comparison of Annual Publications}

The analyzed results of all surveyed articles are MQTT applications with development and implementation. All results of the energy technology are low CPU usage. Some results of the database technology are SQL and NoSQL. In 2015, the majority result of message type is equipment message. All results of platform technology are cloud computing. Most results of the technological industry are healthcare. Many results in 2016 and 2017 are similar. Some results of message type are body message. The minority result of platform technology is fog computing. Some results of the energy technology are support of sleeping clients/multiple gateways/lightweight implementation. Most results of the technological industry are industry 4.0 while the minority is robot. The message type result is mostly equipment message due to growing heterogeneous IoT devices. Fog computing platform is emerging to extend cloud computing at the network edge. MQTT-SN which provides WSN support of more energy savings is also increasing. 


\section{Proposed MQTT Classification}

MQTT classification covers 4 technologies of platform, database, energy and industry as shown in Fig. 2.

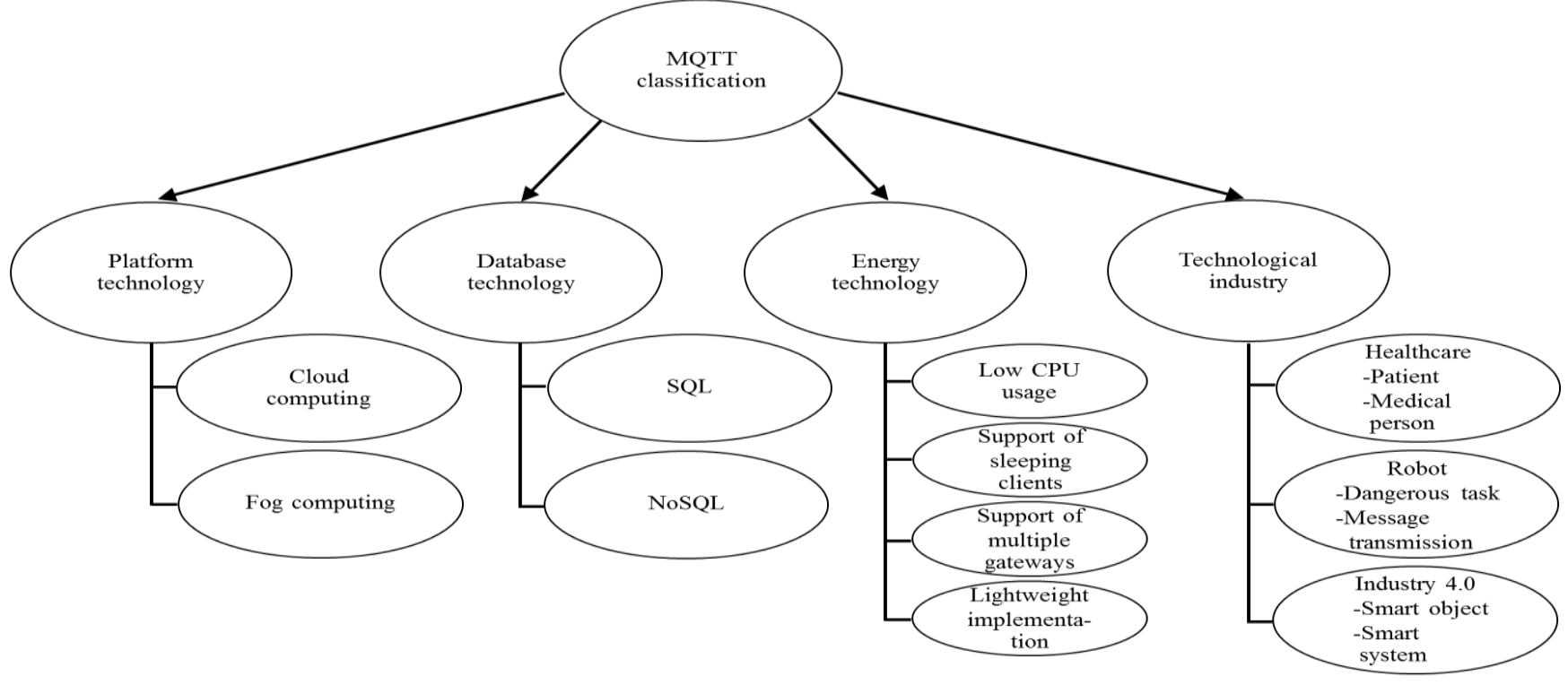

Fig. 2: MQTT classification.

\section{Conclusion}

This paper proposed MQTT classification of technologies. It also presented a survey and analysis of full peer-reviewed MQTT in industries articles between 2011 and 2017 to confirm the classification.

\section{References}

[1] A. Al-Fuqaha, M. Guizani, M. Mohammadi, et al., "Internet of Things: A Survey on Enabling Technologies, Protocols, and Applications," IEEE Communications Surveys and Tutorials, vol. 17, pp. 2347-2376, June 2015.

[2] G. Atsali, S. Panagiotakis, E. Markakis, et al., "A mixed reality 3D system for the integration of X3DoM graphics with real-time IoT data," Multimedia Tools and Applications, vol. 2017, pp. 1-22, June 2017.

[3] D. Barata, G. Louzada, A. Carreiro, et al., "System of acquisition, transmission, storage and visualization of pulse oximeter and ECG data using android and MQTT," Procedia Technology, vol. 9, pp. 1265-1272, December 2013.

[4] P. Bellavista, C. Giannelli, and R. Zamagna, "The PeRvasive Environment Sensing and Sharing Solution," Sustainability, vol. 9, pp. 1-17, April 2017.

[5] O. Belmonte-Fernandez, A. Puertas-Cabedo, J. Torres-Sospedra, et al., "An Indoor Positioning System Based on Wearables for Ambient-Assisted Living," Sensors, vol. 17, pp. 1-22, December 2017.

[6] M. D. Benedetti, F. Messina, G. Pappalardo, et al., "Web-based Simulations of Multi-agent Systems," Simulation: Transactions of the Society for Modeling and Simulation International, vol. 93, pp. 737-748, May 2017.

[7] B.-C. Chifor, I. Bica, and V.-V. Patriciu, "Sensing service architecture for smart cities using social network platforms," Soft Computing, vol. 2017, pp. 4513-4522, February 2017.

[8] R. Dhall and V. Solanki, "An IoT Based Predictive Connected Car Maintenance Approach," International Journal of Interactive Multimedia and Artificial Intelligence, vol. 4, pp. 16-22, March 2017.

[9] P. Diogo, N. V. Lopes, and L. P. Reis, "An ideal IoT solution for real-time web monitoring," Cluster Computing, vol. 20, pp. 2193-2209, May 2017. 
[10] K. Fysarakis, O. Soultatos, C. Manifavas, et al., "XSACd-Cross-domain resource sharing \& access control for smart environments," Future Generation Computer Systems, vol. 2016, pp. 1-11, June 2016.

[11] A. Garcia-de-Prado, G. Ortiz, and J. Boubeta-Puig, "COLLECT: COLLaborativE ConText-aware service oriented architecture for intelligent decision-making in the Internet of Things," Expert Systems With Applications vol. 85, pp. 231-248, May 2017.

[12] G. Gardasevic, M. Veletic, N. Maletic, et al., "The IoT Architectural Framework, Design Issues and Application Domains," Wireless Pers Comm, vol. 92, pp. 127-148, January 2017.

[13] S. Herle and J. Blankenbach, "Enhancing the OGC WPS interface with GeoPipes support for real-time geoprocessing," International Journal of Digital Earth, pp. 1-16, May 2017.

[14] L. Hou, S. Zhao, X. Xiong, et al., "Internet of Things Cloud: Architecture and Implementation," IEEE Communications Magazine, vol. 54, pp. 1-19, December 2016.

[15] U. Hunkeler, H. Truong, and A. Stanford-Clark, "MQTT-S-A publish/subscribe protocol for wireless sensor networks," presented at the Inter Conf on Comm Sys Software \& Middleware Workshops, India, January 6-10, 2008.

[16] D. Husejnagić and A. Sluga, "A conceptual framework for a ubiquitous autonomous work system in the Engineer-ToOrder environment," Inter J of Advanced Manufacturing Technology, vol. 78, pp. 1971-1988, January 2015.

[17] H. Inomoto, S. Saiki, and M. Nakamura, "Design and evaluation of mission-oriented sensing platform with military analogy," International Journal of Pervasive Computing and Communications, vol. 13, pp. 76-91, February 2017.

[18] J. Jin, X. Ma, and I. Kosonen, "An intelligent control system for traffic lights with simulation-based evaluation," Control Engineering Practice vol. 58, pp. 24-33, January 2017.

[19] W. Joe, J. Lee, and K. Jeong, "CSN: The Conceptually Manageable Sensor Network," International Journal of Distributed Sensor Networks, vol. 2015, pp. 1-17, February 2015.

[20] R. Kazala, A. Taneva, M. Petrov, et al., "Wireless network for mobile robot applications," IFAC-PapersOnLine, vol. 48, pp. 231-236, December 2015.

[21] J. Klemets and K. D. Moor, "Patient responsibility reallocation: a user-centered approach to support nurses' handling of nurse calls," Personal and Ubiquitous Computing, vol. 2015, pp. 601-621, June 2015.

[22] N. Leavitt, "Will NoSQL databases live up to their promise," IEEE Computer, vol. 43, pp. 1-3, January 2010.

[23] P. Masek, J. Hosek, K. Zeman, et al., "Implementation of True IoT Vision: Survey on Enabling Protocols and HandsOn Experience," Inter J of Distributed Sensor Networks, vol. 2016, pp. 1-18, February 2016.

[24] M. A. Prada, P. Reguera, S. Alonso, et al., "Communication with resource-constrained devices through MQTT for control education," IFAC-PapersOnLine, vol. 49, pp. 150-155, July 2016.

[25] T. Rault, A. Bouabdallah, and Y. Challal, "Energy efficiency in wireless sensor networks: A top-down survey," Computer Networks, vol. 67, pp. 104-122, April 2014.

[26] R. N. Rodrigues, A. V. C. , G. M. S. , et al., "Publish-Subscribe Architecture for Delivering Assistance to Visually Impaired People," IFAC-PapersOnLine, vol. 49, pp. 150-155, December 2016.

[27] P. Sethi and S. R. Sarangi, "Internet of Things: Architectures, Protocols, and Applications," Journal of Electrical and Computer Engineering, vol. 2017, pp. 1-25, January 2017.

[28] A. d. Silva, R. Ohta, M. d. Santos, et al., "A Cloud-based architecture for the Internet of Things targeing industrial devices remote monitoring and control," IFAC-PapersOnLine, vol. 49, pp. 108-113, December 2016.

[29] A. Talaminos-Barroso, M. A. Estudillo-Valderrama, L. M. Roa, et al., "A Machine-to-Machine protocol benchmark for eHealth applications," Computer Methods and Programs in Biomedicine, vol. 129, pp. 1-11, June 2016.

[30] L. Treyer, B. Klein, R. Konig, et al., "Lightweight urban computation interchange (LUCI): a system to couple heterogeneous simulations and views," Spatial Information Research, vol. 24, pp. 291-302, May 2016. 
[31] N. Verba, K.-M. Chao, A. James, et al., "Platform as a service gateway for the Fog of Things," Advanced Engineering Informatics, vol. 2016, pp. 1-15, November 2016.

[32] G. Villarrubia, D. Hernandez, J. Paz, et al., "Combination of multi-agent systems and embedded hardware for the monitoring and analysis of diuresis," Inter J of Distributed Sensor Networks, vol. 13, pp. 1-17, July 2017.

[33] Z. Yang, Q. H. Zhou, L. Lei, et al., "An IoT-cloud Based Wearable ECG Monitoring System for Smart Healthcare," Journal of Medical Systems, vol. 40, pp. 1-11, October 2016.

[34] A. M. Zambrano, I. Perez, C. Palau, et al., "Technologies of Internet of Things applied to an Earthquake Early Warning System," Future Generation Computer Systems, vol. 75, pp. 206-215, October 2017.

[35] E. d. S. Zancul, S. M. Takey, A. P. B. Barquet, et al., "Business process support for IoT based product-service systems (PSS)," Business Process Management Journal, vol. 22, pp. 305-323, November 2016.

[36] D. Zubov, "An Energy-Saving Concept of the Smart Building Power Grid with Separated Lines for Standby Devices," Brain-Broad Research in Artificial Intelligence and Neuroscience, vol. 7, pp. 148-156, August 2016. 